\title{
Class-preserving automorphisms of some finite $p$-groups
}

\author{
Mahak Sharma \\ Department of Applied Sciences and Humanities \\ Baddi University of Emerging Sciences and Technology, Baddi, India \\ Deepak Gumber* \\ School of Mathematics and Computer Applications \\ Thapar University, Patiala - 147004 , India
}

\begin{abstract}
Let $G$ be a finite $p$-group of order $p^{5}$, where $p$ is a prime. We give necessary and sufficient conditions on $G$ such that $G$ has a non-inner class-preserving automorphism. As a consequence, we give short and alternate proofs of results of section 5 of Yadav [Proc. Indian Acad. Sci. (Math. Sci.) 118 (2008), 1-11] and Theorem 4.2 of Kalra and Gumber [Indian J. Pure Appl. Math. 44 (2013), 711-725].
\end{abstract}

2010 Mathematics Subject Classification: 20D45, $20 \mathrm{D} 15$.

Keywords: Class-preserving automorphism, p-group, Central automorphism.

1 Introduction Let $G$ be a finite group. An automorphism $\alpha$ of $G$ is called a classpreserving automorphism if for each element $x \in G$, there exists an element $g_{x} \in G$ such that $\alpha(x)=g_{x}^{-1} x g_{x}$; and is called an inner automorphism if for all $x \in G$, there exists a fix element $g \in G$ such that $\alpha(x)=g^{-1} x g$. The group $\operatorname{Inn}(G)$ of all inner automorphisms of $G$ is a normal subgroup of the group $\operatorname{Aut}_{c}(G)$ of all class-preserving automorphisms of $G$. We denote the group $\operatorname{Aut}_{c}(G) / \operatorname{Inn}(G)$ of all class-preserving outer automorphisms by $\mathrm{Out}_{c}(G)$. The interest in class-preserving automorphisms took place when Burnside 2, p. 463] asked the question: Does there exist any finite group $G$ such that $G$ has a noninner class-preserving automorphism? Burnside 3 himself gave an affirmative answer to his question by constructing a group $G$ of order $p^{6}$ for which $\operatorname{Out}_{c}(G)$ was non-trivial. For more details about this problem, one can see the survey article by Yadav [13].

In this note, we are especially interested in class-preserving automorphisms of $p$-groups of order upto $p^{5}$. Using the known classifications and presentations of finite extra-special $p$-groups and of groups of order $p^{4}$, Kumar and Vermani [8, 9, proved that if $G$ is an extraspecial $p$-group (in particular if $|G|=p^{3}$ ) or if $|G|=p^{4}$, then $\operatorname{Out}_{c}(G)=1$. It follows from [6] that all finite $p$-groups of order $p^{5}$, where $p$ is an odd prime, are partitioned into ten isoclinism families; and from 4 that all finite 2 -groups of order $2^{5}$ are partitioned into eight isoclinism families. Yadav [12] proved that if $G$ and $H$ are two finite non-abelian isoclinic groups, then $\operatorname{Aut}_{c}(G) \simeq \operatorname{Aut}_{c}(H)$. He then showed, by picking up one group from each isoclinism family, that $\operatorname{Out}_{c}(G) \neq 1$ for the groups $\Phi_{7}\left(1^{5}\right)$ and $\Phi_{10}\left(1^{5}\right)$ from seventh and tenth family in [6], and hence concluded that if $G$ is a finite $p$-group of order $p^{5}$, where $p$ is an odd prime, then $\operatorname{Out}_{c}(G) \neq 1$ if and only if $G$ is isoclinic to a group either in the seventh or in the tenth family. Recently, Kalra and Gumber [7, Theorem 4.2], using the classifications, isoclinism families and presentations given by Hall and Senior [4] and Sag

* Research supported by University Grants Commission of India under the Research Award Scheme 
and Wamsley [10, have shown that if $G$ is a finite 2-group of order $2^{5}$, then $\operatorname{Out}_{c}(G)=1$ except for the forty fourth and forty fifth groups from the sixth family in 4 .

Hertweck [5, Proposition 14.4] proved that if $G$ is a finite group having an abelian normal subgroup $A$ with cyclic quotient $G / A$, then class-preserving automorphisms of $G$ are inner automorphisms. Yadav [12, Corollary 3.6] proved that if $G$ is a finite $p$-group of nilpotence class 2 such that $G^{\prime}$ is cyclic, then $\operatorname{Out}_{c}(G)=1$. We shall use these results quite frequently in our proofs. Observe that if $G$ is an extra-special p-group, then $G^{\prime}$ is cyclic; and if $|G|=p^{4}$, then $G$ has a maximal abelian subgroup. It follows that for these groups $G$, $\operatorname{Out}_{c}(G)=1$. The present note is the result of an effort to find necessary and sufficient conditions, without using the available classifications and presentations, on a finite $p$-group $G$ for which $\operatorname{Out}_{c}(G)$ is non-trivial. In section 2, we prove our main theorem, Theorem 2.3 , which gives the necessary and sufficient conditions on a finite $p$-group $G$ of order $p^{5}$ for which $\operatorname{Out}_{c}(G) \neq 1$. As a consequence, we obtain short and alternate proofs of the results of Yadav [12, Section 5] and Kalra and Gumber [7, Theorem 4.2].

An automorphism $\alpha$ of a group $G$ is called a central automorphism if it induces the identity automorphism on $G / Z(G)$; or equivalently, $x^{-1} \alpha(x) \in Z(G)$ for all $x \in G$. By $\operatorname{Aut}_{z}(G)$ we denote the group of all central automorphisms of $G$. For $x \in G, x^{G}$ denotes the conjugacy class of $x$ in $G$. By $G^{\prime}$ and $\Phi(G)$, we respectively denote the commutator and the Frattini subgroup of G. A cyclic group of order $m$ is denoted as $C_{m}$ and $\gamma_{3}(G)$ denotes the third term of the lower central series of $G$. The symbol $c l(G)$ denotes the nilpotence class of $G$ and by $d(G)$ we denote the smallest cardinality of a generating set of $G$. If $H$ is a non-trivial proper normal subgroup of $G$, then $(G, H)$ is called a Camina pair if and only if $H \subseteq[x, G]$ for all $x \in G-H$, where $[x, G]=\{[x, g] \mid g \in G\}$.

2 Proof of Theorem. We start with the following crucial technical lemma. The lemma can be of independent interest also.

Lemma 2.1 Let $G$ be any group such that $\operatorname{Out}_{c}(G / Z(G))$ is trivial. Then $\operatorname{Aut}_{c}(G)=$ $\left(\operatorname{Aut}_{c}(G) \cap \operatorname{Aut}_{z}(G)\right) \operatorname{Inn}(G)$. In addition, if $G$ is finite, then

$$
\left|\operatorname{Aut}_{c}(G)\right|=\frac{\left|\operatorname{Aut}_{c}(G) \cap \operatorname{Aut}_{z}(G)\right||\operatorname{Inn}(G)|}{|Z(\operatorname{Inn}(G))|} .
$$

Proof. If $\alpha \in \operatorname{Aut}_{c}(G)$, then it induces a class-preserving automorphism, say $\bar{\alpha}$, on $G / Z(G)$ given by $\bar{\alpha}(x Z(G))=\alpha(x) Z(G)$ for all $x \in G$. Since $\operatorname{Out}_{c}(G / Z(G))=1, \alpha(x) Z(G)=$ $a^{-1} x a Z(G)$ for a fix $a \in G$. Therefore, for each $x \in G$, there exists an element $z_{x} \in Z(G)$ such that $\alpha(x)=a^{-1} x a z_{x}=a^{-1}\left(x z_{x}\right) a$. Define a map $\beta: G \rightarrow G$ by $\beta(x)=a \alpha(x) a^{-1}$ for all $x \in G$. It is easy to see that $\beta \in \operatorname{Aut}_{c}(G) \cap \operatorname{Aut}_{z}(G)$ and $\alpha=i_{a} \beta$, where $i_{a}$ denotes the inner automorphism of $G$ given by conjugation with $a$. It thus follows that $\operatorname{Aut}_{c}(G)=\left(\operatorname{Aut}_{c}(G) \cap \operatorname{Aut}_{z}(G)\right) \operatorname{Inn}(G)$.

Proposition 2.2 Let $G$ be a finite non-abelian p-group of order $p^{5}$ such that $|Z(G)| \geq p^{2}$. Then $\operatorname{Out}_{c}(G)=1$.

Proof. If $|Z(G)| \geq p^{3}$, then $G$ has a maximal abelian subgroup and hence $\operatorname{Out}_{c}(G)=1$. We therefore suppose that $|Z(G)|=p^{2}$. Then $\operatorname{cl}(G)$ is either 2 or 3. First suppose that $c l(G)=2$. Then $G^{\prime} \leq Z(G)$. If $G^{\prime}$ is cyclic, then $\operatorname{Out}_{c}(G)=1$. Therefore suppose that $G^{\prime}=Z(G) \simeq C_{p} \times C_{p}$. Then $\exp (G / Z(G))=\exp \left(G^{\prime}\right)=p$. It follows that $Z(G)=G^{\prime}=$ $\Phi(G)$ and hence $d(G)=3$. If $G=\langle a, b, c\rangle$, then $G^{\prime}=\langle[a, b],[a, c],[b, c]\rangle$. We can assume that $[b, c]=[a, b]^{m}[a, c]^{n}$ for some $m, n, 0 \leq m, n \leq p-1$. Set $u:=b a^{-n}$ and $v:=c a^{m}$. Then

$$
[u, v]=\left[b a^{-n}, c a^{m}\right]=[b, c][b, a]^{m}[a, c]^{-n}=1 .
$$

It follows that $\left\langle u, v, G^{\prime}\right\rangle$ is a maximal abelian subgroup of $G$ and hence $\operatorname{Out}_{c}(G)=1$. 
Now suppose that $\operatorname{cl}(G)=3$. Then $G^{\prime}$ is abelian of order $p^{2}$ or $p^{3}$. First assume that $\left|G^{\prime}\right|=p^{2}$. Since $G / C_{G}\left(G^{\prime}\right)$ is isomorphic to a subgroup of $\operatorname{Aut}\left(G^{\prime}\right),\left[G: C_{G}\left(G^{\prime}\right)\right] \leq p$ and hence $\left|C_{G}\left(G^{\prime}\right)\right|=p^{4}$. The subgroup $G^{\prime} Z(G)$ is of order $p^{3}$ and is contained in $C_{G}\left(G^{\prime}\right)$. It follows that $C_{G}\left(G^{\prime}\right)=\left\langle a, G^{\prime} Z(G)\right\rangle$, where $a \in C_{G}\left(G^{\prime}\right)-G^{\prime} Z(G)$, is a maximal abelian subgroup of $G$ and hence $\operatorname{Out}_{c}(G)=1$. Next assume that $\left|G^{\prime}\right|=p^{3}$. Then $G^{\prime}=\Phi(G)$ and $d(G)=2$. Let $G=\langle a, b\rangle$ and let $w:=[a, b], u:=[a, w]$ and $v:=[b, w]$. Then $G^{\prime}=\left\langle w, \gamma_{3}(G)\right\rangle$ and $\gamma_{3}(G)=\langle u, v\rangle$. Since $[a, b]^{p} \equiv\left[a, b^{p}\right] \equiv 1 \quad\left(\bmod \gamma_{3}(G)\right),\left|G^{\prime} / \gamma_{3}(G)\right|=p$ and hence $\left|\gamma_{3}(G)\right|=p^{2}$. If $\left|h^{G}\right|=p$ for some $h \in G-G^{\prime}$, then $C_{G}(h)=\left\langle h, G^{\prime}\right\rangle$ is a maximal abelian subgroup of $G$ and thus $\operatorname{Out}_{c}(G)=1$; and if $\left|h^{G}\right|=p^{3}$ for some $h \in G-G^{\prime}$, then $\left|C_{G}(h)\right|=p^{2}$ and hence $h \in Z(G)$, which is absurd. We therefore suppose that $\left|x^{G}\right|=p^{2}$ for all $x \in G-G^{\prime}$. Then $\left|\operatorname{Aut}_{c}(G)\right| \leq p^{4}$. Let $\left|\operatorname{Aut}_{c}(G)\right|=p^{4}$. Then, for any $x, y \in G$, there exists an $\alpha \in \operatorname{Aut}_{c}(G)$ such that $\alpha(a)=x^{-1} a x$ and $\alpha(b)=y^{-1} b y$. In particular, there exists an $\alpha \in \operatorname{Aut}_{c}(G)$ such that $\alpha(a)=b^{-1} a b$ and $\alpha(b)=a^{-1} b a$. Now $(a b)^{-1} \alpha(a b) \in[a b, G]$. But $(a b)^{-1} \alpha(a b)=b^{-1} a^{-1} \alpha(a) \alpha(b)=b^{-1} w b w^{-1}=[w, b]=v^{-1}$. Thus $v^{-1} \in[a b, G]$. Let $v^{-1}=[a b, h]$, where $h \in G$. Observe that $u v=[a, w][b, w]=[a b, w]$ and therefore $u=(u v) v^{-1}=[a b, w][a b, h]=[a b, w h] \in[a b, G]$. Thus $\gamma_{3}(G) \leq[a b, G]$. But since $|[a b, G]|=\left|(a b)^{G}\right|=p^{2}, \gamma_{3}(G)=[a b, G]$. Thus $w^{-1}=[b, a]=[a b, a] \in[a b, G]=\gamma_{3}(G)$. This is a contradiction and hence $\operatorname{Out}_{c}(G)=1$. This completes the proof.

Theorem 2.3 Let $G$ be a finite non-abelian p-group of order $p^{5}$. Then $\operatorname{Out}_{c}(G) \neq 1$ if and only if $|Z(G)|=p, Z(G)<G^{\prime}$ and either $(i) \operatorname{cl}(G)=3$ and $d(G)=3$ or (ii) $\operatorname{cl}(G)=4$ and $Z(G) \subseteq[x, G]$ for all $x \in G-G^{\prime}$.

Proof. First suppose that $\mathrm{O} u t_{c}(G) \neq 1$. Then $|Z(G)|=p$ by Proposition 2.2 and hence $Z(G)<G^{\prime}$. It follows that $G$ is purely non-abelian and $c l(G)$ is either 3 or 4 . Suppose that $c l(G)=3$. Since $\left|G^{\prime}\right|=p^{2}$ or $p^{3},\left|\operatorname{Aut}_{z}(G)\right|=\left|\operatorname{Hom}\left(G / G^{\prime}, Z(G)\right)\right| \leq p^{3}$ by [1, Theorem 1]. Also, since $G / Z(G)$ is a class 2 group of order $p^{4},|Z(\operatorname{Inn}(G))|=p^{2}$. It now follows by Lemma 2.1 that

$$
\left|\operatorname{Aut}_{c}(G)\right|=p^{2}\left|\operatorname{Aut}_{c}(G) \cap \operatorname{Aut}_{z}(G)\right| \leq p^{5} .
$$

Since $\left|\operatorname{Aut}_{c}(G)\right|>|\operatorname{Inn}(G)|=p^{4},\left|\operatorname{Aut}_{z}(G)\right|=p^{3}$ and hence $d(G)=3$. Next suppose that $c l(G)=4$. Then $G$ is of maximal class and hence $\left|G^{\prime}\right|=p^{3},|Z(\operatorname{Inn}(G))|=p, d(G)=2$ and $\left|\operatorname{Aut}_{z}(G)\right|=\left|\operatorname{Hom}\left(C_{p} \times C_{p}, C_{p}\right)\right|=p^{2}$. Thus

$$
\left|\operatorname{Aut}_{c}(G)\right|=p^{3}\left|\operatorname{Aut}_{c}(G) \cap \operatorname{Aut}_{z}(G)\right| \leq p^{5},
$$

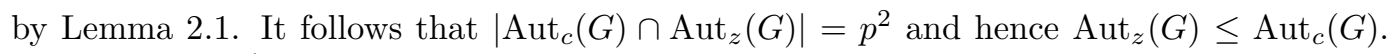
Let $a \in G-G^{\prime}$ and let $G=\langle a, b\rangle$. Suppose that all central automorphisms of $G$ fix $a$. Then each $1 \neq \alpha \in \operatorname{Aut}_{z}(G)$ would have to move $b$. Since $\left|\operatorname{Aut}_{z}(G)\right|=p^{2}$, this would require that $|Z(G)|=p^{2}$, which is not so. Thus, there exists a central automorphism $\alpha$ such that $\alpha(a)=a z$ for some $1 \neq z \in Z(G)$. On the other hand, since $\alpha$ is class-preserving, $\alpha(a)=g^{-1} a g$ for some $g \in G$. It follows that $z=[a, g]$ and, since $z^{n}=\left[a, g^{n}\right]$ for all $n \geq 1$, $Z(G) \subseteq[x, G]$ for all $x \in G-G^{\prime}$.

To prove the converse, we first suppose that $|Z(G)|=p, Z(G)<G^{\prime}, \operatorname{cl}(G)=4$ and $Z(G) \subseteq[x, G]$ for all $x \in G-G^{\prime}$. Since $G$ is of maximal class, $|Z(\operatorname{Inn}(G))|=p,\left|G^{\prime}\right|=p^{3}$, $d(G)=2$ and hence $\left|\operatorname{Aut}_{z}(G)\right|=\left|\operatorname{Hom}\left(C_{p} \times C_{p}, C_{p}\right)\right|=p^{2}$. Also, since $Z(G) \subseteq[x, G]$ for all $x \in G-G^{\prime}, \operatorname{Aut}_{z}(G) \leq \operatorname{Aut}_{c}(G)$. It follows by Lemma 2.1 that $\left|\operatorname{Aut}_{c}(G)\right|=p^{5}>|\operatorname{Inn}(G)|$. Next suppose that $|Z(G)|=p, Z(G)<G^{\prime}$ and $c l(G)=d(G)=3$. Then $\left|G^{\prime}\right|=|\Phi(G)|=p^{2}$ and hence $\left|\operatorname{Aut}_{z}(G)\right|=\left|\operatorname{Hom}\left(G / G^{\prime}, Z(G)\right)\right|=p^{3}$. It follows from [11, Theorems 4.7 and 5.1] that $(G, Z(G))$ is a Camina pair. Thus $Z(G) \subseteq[x, G]$ for all $x \in G-Z(G)$ and hence $\operatorname{Aut}_{z}(G) \leq \operatorname{Aut}_{c}(G)$. Since $|G / Z(G)|=p^{4}$ and $c l(G / Z(G))=2,|Z(\operatorname{Inn}(G))|=p^{2}$ and thus $\left|\operatorname{Aut}_{c}(G)\right|=p^{5}>|\operatorname{Inn}(G)|$ by Lemma 2.1. This proves the theorem. 
As a consequence of Theorem 2.3, we can now obtain the following results of Yadav 12 , Section 5] and Kalra and Gumber [7, Theorem 4.2]. In [6], the groups of order $p^{5}$, where $p$ is an odd prime, are divided into ten isoclinism families; and in 4, the groups of order 32 are divided into eight isoclinism families. The isoclinism families appearing in Corollary 2.4 and Corollary 2.5 are respectively from [6] and [4]. The $i$-th family is denoted as $\Phi_{i}$. We would like to remark here that the derived groups of all the groups in the tenth family of [6] are elementary abelian for $p \geq 5$, but, for $p=3$, the derived groups are not elementary abelian because $\left|\alpha_{2}\right|=9$. It follows that, for $p=3$, the groups $H_{1}=\Phi_{10}(2111) a_{0}, H_{2}=\Phi_{10}(2111) a_{1}$ and $H=\Phi_{10}\left(1^{5}\right)$ are not in the tenth family.

Corollary 2.4 Let $G$ be a finite p-group of order $p^{5}$, where $p$ is an odd prime. Then $\operatorname{Out}_{c}(G) \neq 1$ if and only if $G$ is isomorphic to one of the groups in $\Phi_{7}$ or one of the groups in $\Phi_{10}$ for $p \geq 5$ or to $H, H_{1}$ or $H_{2}$.

Proof. If $G$ is any group from the first six families, then either $c l(G)<3$ or $|Z(G)|>p$. There are two isoclinism families $\Phi_{7}$ and $\Phi_{8}$ consisting of groups of class 3 ; and there are two isoclinism families $\Phi_{9}$ and $\Phi_{10}$ consisting of groups of class 4 . The only group in eighth family is $\Phi_{8}(32)$ with $d\left(\Phi_{8}(32)\right)=2$. Any group $G$ in the seventh family is generated by $\alpha, \alpha_{1}, \beta$, and it is easy to see that the $p$-th power of any of these generators is either 1 or is in $G^{\prime}$. It thus follows that $|\Phi(G)|=\left|G^{\prime}\right|=p^{2}$ and consequently $d(G)=3$. Any group $G$ in the ninth family is minimally generated by $\alpha$ and $\alpha_{1}$ with abelian commutator subgroup $G^{\prime}=\left\langle\left[\alpha_{1}, \alpha\right], \gamma_{3}(G)\right\rangle=\left\langle\alpha_{2}, \gamma_{3}(G)\right\rangle$ and $\gamma_{3}(G)=\left\langle\left[\alpha_{1}, \alpha_{2}\right],\left[\alpha, \alpha_{2}\right], Z(G)\right\rangle=\left\langle\alpha_{3}^{-1}, Z(G)\right\rangle$. Thus $\alpha_{1}$ commutes with $G^{\prime}$ and hence $\left|\alpha_{1}^{G}\right|=p$. It is easy to see that any element $g$ of $G$ is of the form $g^{\prime} \alpha_{1}^{k} \alpha^{j}$, where $0 \leq j, k \leq p-1$ and $g^{\prime} \in G^{\prime}$, and hence $\left[\alpha_{1}, g\right]=\left[\alpha_{1}, g^{\prime} \alpha_{1}^{k} \alpha^{j}\right]=$ $\left[\alpha_{1}, \alpha^{j}\right] \in G^{\prime}-\gamma_{3}(G)$. It follows that $Z(G)$ is not contained in $[x, G]$ for all $x \in G-G^{\prime}$. Let $G$ be any group in the tenth family. Any element $g \in G-G^{\prime}$ is of the form $g=g^{\prime} \alpha_{1}^{l} \alpha^{m}$, where $0 \leq l, m \leq p-1$ and $g^{\prime} \in G^{\prime}$. If $m \neq 0$, then $\left[g, \alpha_{3}\right]=\left[\alpha^{m}, \alpha_{3}\right] \in Z(G)$. Let $m=0$ and $l \neq 0$. It is easy to see, by induction, that $\left[\alpha_{1}^{l}, \alpha_{2}\right]=\left[\alpha_{1}, \alpha_{2}\right]^{l} z_{1} z_{2} \ldots z_{l-1}$, where $z_{1}, z_{2}, \ldots, z_{l-1} \in Z(G)$. Then $\left[g, \alpha_{2}\right]=\left[g^{\prime} \alpha_{1}^{l}, \alpha_{2}\right]=\left[\alpha_{1}^{l}, \alpha_{2}\right]=\left[\alpha_{1}, \alpha_{2}\right]^{l} \in Z(G)$, because $\left[\alpha_{1}, \alpha_{2}\right] \in Z(G)$. It follows that $Z(G) \subseteq[x, G]$ for all $x \in G-G^{\prime}$.

There are, in all, fifty one groups of order 32. Sag and Wamsley [10 have given minimal presentations of these groups. As mentioned in [10], the groups are in the same order in [4] and [10. We denote the $i$-th group as $G_{i}$.

Corollary 2.5 Let $G$ be a finite group of order 32 . Then $\operatorname{Out}_{c}(G) \neq 1$ if and only if either $G$ is isomorphic to $G_{44}$ or isomorphic to $G_{45}$.

Proof. First forty three groups are divided into first five families. The families $\Phi_{1}, \Phi_{2}, \Phi_{4}$ and $\Phi_{5}$ contain groups of class $\leq 2$. The third family contains ten groups $G_{23}-G_{32}$ of class 3 , and each of these groups has center of order 4 . The sixth family contains groups $G_{44}$ and $G_{45}$. Both of these groups are of class 3, rank 3, and with centers of order 2. The seventh family contains groups $G_{46}-G_{48}$ of class 3 and rank 2 . The last family contains 2-generated groups $G_{49}-G_{51}$ of maximal class. It is clear from [10] that in each of these groups, one generator, say $x$, is of order 16 and hence $\left|x^{G}\right|=2$. If $y$ is another generator, then $y^{-1} x y$ is respectively $x^{15}, x^{7}$ and $x^{15}$ in the group $G_{49}, G_{50}$ and $G_{51}$. The center of each of these groups is $\left\{1, x^{8}\right\}$. For any element $g$ in these groups, if $[x, g]=x^{8}$, then $g^{-1} x g=x^{9}$, which is not so.

\section{References}

[1] J. E. Adney and T. Yen, Automorphisms of a p-group, Illinois J. Math. 9 (1965), 137143. 
[2] W. Burnside, Theory of groups of finite order, 2nd Ed. Dover Publication, Inc., 1955. Reprint of the 2nd edition (Cambridge, 1911).

[3] W. Burnside, On the outer automorphisms of a group, Proc. London Math. Soc. 11 (1913), 40-42.

[4] M. Hall Jr. and J. K. Senior, The groups of order $2^{n}(n \leq 6)$, Macmillan, New York, 1964.

[5] M. Hertweck, Contributions to the integral representation theory of groups, Habilitationsschrift (University of Stuttgart) (2004), available at http://elib.unistuttgart.de/opus/volltexte/2004/1638

[6] R. James, The groups of order $p^{6}$ (p an odd prime), Math. Comp. 34 (1980), 613-637.

[7] H. Kalra and D. Gumber, On equality of central and class preserving automorphisms of finite p-groups, Indian J. Pure Appl. Math. 44 (2013), 711-725.

[8] M. Kumar and L. R. Vermani, Hasse principle for extra-special p-groups, Proc. Japan Acad. 76 (2000), 123-125.

[9] M. Kumar and L. R. Vermani, Hasse principle for groups of order $p^{4}$, Proc. Japan Acad. 77 (2001), 95-98.

[10] T. W. Sag and J. W. Wamsley, Minimal presentations for groups of order $2^{n}, n \leq 6, \mathrm{~J}$. Aust. Math. Soc. 15 (1973), 461-469.

[11] H. Tandra and W. Moran, Flatness conditions on finite p-groups, Comm. Algebra. 32 (2004), 2215-2224.

[12] M. K. Yadav, On automorphisms of some finite p-groups, Proc. Indian Acad. Sci. (Math. Sci.) 118 (2008), 1-11.

[13] M. K. Yadav, Class preserving automorphisms of finite p-groups-A Survey, Groups St. Andrews, LMS Lecture Note Series 388, Vol. 2 (2011), 569-579. 